Corresponding Author:

Azadeh karami Robati;

Department of Pathobiology,

Science and Research

Branch, Islamic Azad

University, Tehran, Iran

Phone: +989132978745

email:

karamy8926@yahoo.com

Production and Hosting by

Knowledge E

(c) Mohammad Khalili

et al. This article is distributed under the terms of the

Creative Commons

Attribution License, which

permits unrestricted use and redistribution provided that the original author and source are credited.

Editor-in-Chief:

Dr. Alireza Rafiei
Research Article

\section{Subtilisin Genes (SUB1-3) Presence in Microsporum canis Isolates with Human and Animal Source}

\author{
Mohammad Khalilili, Mansour Bayat ${ }^{2}$, Seyed Jamal Hashemi Hazaveh ${ }^{3}$, and \\ Azadeh karami Robati ${ }^{2}$ \\ ${ }^{1}$ Faculty of Veterinary Medicine, Department of pathobiology, Shahid Bahonar University of \\ Kerman, Kerman, Iran \\ ${ }^{2}$ Department of Pathobiology, Science and Research Branch, Islamic Azad University, Tehran, \\ Iran \\ ${ }^{3}$ Department of Mycology and Parasitology, Tehran University of Medical Sciences, Tehran, Iran
}

\section{Abstract}

Background: The presence of subtilisin genes (SUBs) coding for serine proteases in Microsporum canis DNA, contribute to the adherence of fungi to keratinized tissues. The aim of this study was identifed the presence/absence of subtilisin gene family in M.canis isolated from human and animal source.

Materials and Methods: This cross-sectional study was performed on 24 samples of patients and pets with dermatophytosis from September to November 2017. Genomic DNA, pertaining to all pure colonies in Sabouraud agar with cycloheximide and chloramphenicol (SCC) and Sabouraud Dextrose agar(SDA), extracted, using rapid method without liquid nitrogen. M. canis were detected by molecular test. SUB1, SUB2 and SUB3 gene of $M$. canis amplified. The relative frequency of the genomic sequences also calculated.

Results: M. canis DNA were extracted from cats $(n=6)$, dog $(n=1)$ and human $(n=3)$ with tinea corporis and showed a high percentage for SUB2 (90\%; 9/10) and the absence of SUB genes in a $M$. canis isolated from dog with develop dermatophytosis. The same results were observed in the frequency of the SUBs presence from the colonies grown on SCC and SDA.

Conclusion: The presence of SUBs in M. canis isolates with human and animal source will be the basal to understand zoonotic infections. The absence SUBs in clinical isolate, indicates that they are indispensable for initiation of the infection.

Keywords: Microsporum canis, Dermatophytosis, Subtilisin Genes, Human. Animal, PCR

\section{Introduction}

Two important keratinophilic and keratinolytic characteristics of dermatophytes indicate S OPEN ACCESS 
(parasitic stage) [1-3]. Microsporum canis is the main cause of dermatophytosis in cats and humans, the natural reservoir of which is pets [4, 5]. Methods of routine diagnostic mycology laboratory are used to identify dermatophytes, Sabouraud Cycloheximide Chloramphenicol Agar is medium selective for dermatophytes. It is a recommended medium with antibiotics for eliminate other microorganisms [6]. Chloramphenicol and cycloheximide effects on the synthesis of enzymes in eukaryotic cell [7] and in yeast [8]. Present a PCR-based method for identifying $M$. canis, using specific primer and rapid DNA extraction method from clinical samples and isolates, leading to appropriate and faster treatment and infection control [9]. Pathogenic $M$. canis is dermatophyte that has the ability to attack keratinized structures [10]. Study on mechanisms of dermatophyte infection focused mainly on secreted serine protease [11]. A gene family encode subtilisin-like proteases in $M$. canis. The role of secreted subtilisin has been identified, during the infection steps [12]. The aim of this study was identifed the presence/absence of SUB1, SUB2, and SUB3 in M. Canis DNA isolated from human and animal source and effect of cycloheximide and chloramphenicol in Sabouraud agar on subtilisin genes presence.

\section{Materials and Methods}

\subsection{Collection of human and animal samples}

This cross-sectional study was performed on 24 samples of patients and pets with dermatophytosis from september to November 2017, 14 samples of cats and dogs whit dermatophytosis were collected from three veterinary clinics in Kerman. 10 samples of skin lesions were collected from patients with dermatophytosis referred to Tehran's mycological laboratory.

\subsection{Direct microscopic and laboratory examination}

Skin and hair samples were examined by $10 \% \mathrm{KOH}$ and Lacto phenol blue and cultured in Sabouraud agar with cycloheximide $(0.5 \mathrm{~g} / \mathrm{l})$ and chloramphenicol(0.05g/l)(SCC) (Quelab) and Sabouraud Dextrose Agar(SDA)(Quelab) [13]. In two separate groups, colonies were grown of $M$. canis (from human and animal infections) in SCC and SDA cultured in plates of $20 \mathrm{ml}$ Sabouraud Dextrose Broth (Quelab) and incubated to 5 days at $25^{\circ} \mathrm{C}$. The mycelium was grounded with a pestle in a mortar Surrounded by ice pack and without using liquid nitrogen and total DNA extracted by the rapid method [14]. 


\subsection{PCR test for M. canis identification}

The specific primers Mcfor ( $5^{\prime}$ GTGTGATGGACGACCGTCCCCCCT 3 ') and Mcrev (5' ATAATACATGGTGCGTTAGGCCAGCCTG $3^{\prime}$ ) with NCBI Reference Sequence: NW_003299166, were prepared from Humanizing Genomics Macrogen Company [9] (Control positive was strain of M. canis (PTCC: 5069) and control negative was strain of Trichophyton rubrum (ATCC11404).

\subsection{Molecular studies for SUB genes}

The primers were purchased from Humanizing Genomics Macrogen Company based on the nucleotide sequences of the SUB1-3 genes in the GenBank database for $M$. canis [15]. All PCR amplification were performed in a Thermal Cycler (Eppendorf) in a final volume of $25 \mu \mathrm{l}$. PCR cycling consisted of $94^{\circ} \mathrm{C}$ for 5 min followed by 35 cycles of 30 seconds at $94^{\circ} \mathrm{C}, 1 \mathrm{~min}$ at $58-62^{\circ} \mathrm{C}$ and 45 seconds at $72^{\circ} \mathrm{C}$ and a final extension step of 7 min at $72^{\circ} \mathrm{C}$. Each PCR product was electrophoreed on $1 \%$ agarose gel in TBE buffer at $90 \mathrm{~V}$ for $1 \mathrm{~h}[14]$.

\subsection{Statistical analysis}

The relative frequency of subtilisin genes and their frequency distribution among isolates were analyzed by SPSS software version 16 and chi-square test.

\section{Results}

\subsection{Isolation and identification of $M$. canis strains}

Dermatophytic lesions were identified in $58 \%$ of animal samples (14/24), based on microscopic and macroscopic examination. On the other hand, yeast $(n=1)$ and saprophytes $(n=9)$ isolated from fungal infection [10]. M. canis were detected from human ( $n=3)$, cats $(n=6)$ and dog $(n=1)$ whit tinea corporis by observing the fragment 176 bp from PCR products in the $2 \%$ agarose gel (Fig. 1).

\subsection{Identification of SUB1-3 genes}

Positive results of PCR products were observed different fragment of SUB1(1540bp), SUB2(1496bp), and SUB3(1388bp), indicated the presence of subtilisin virulence genes 


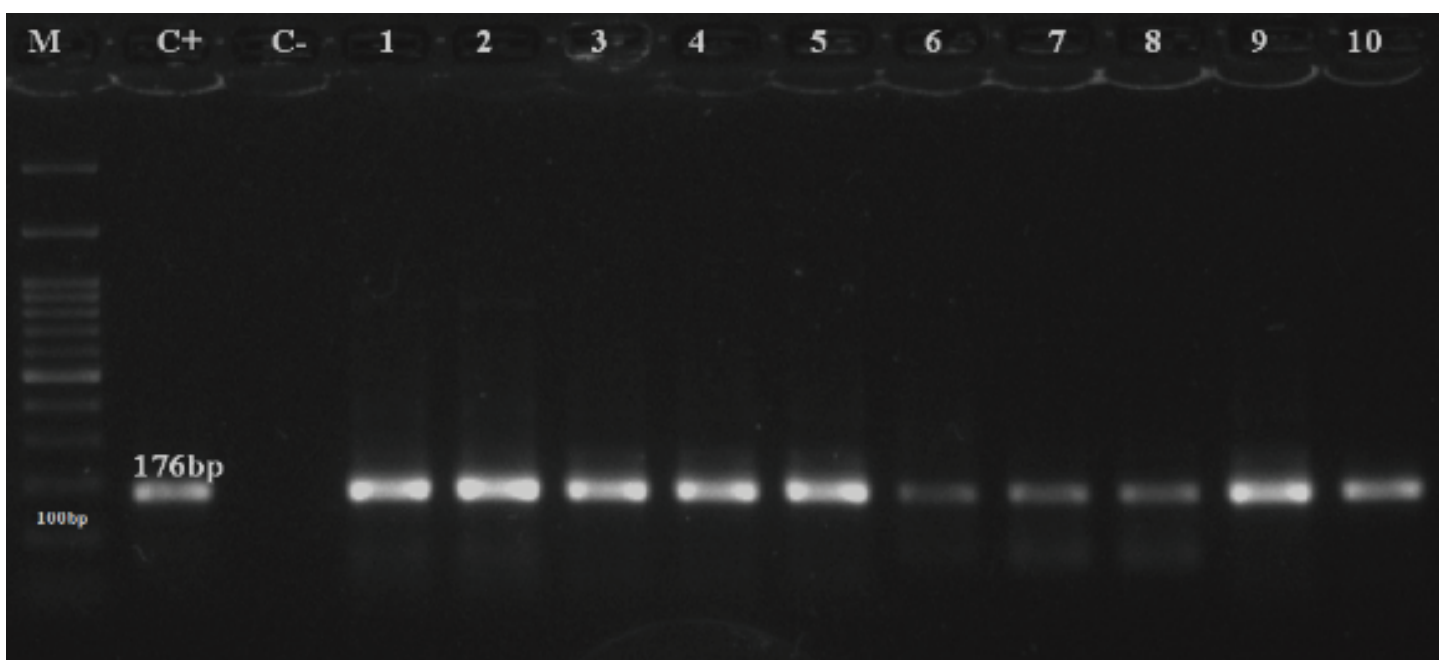

Figure 1: Positive results of $176 \mathrm{bp}$ fragment of ITS1 sequence of the ribosomal DNA were obtained for 10/10 samples containing $M$. canis; Lane $\mathrm{M}-100-3000$ bp DNA molecular weight markers. Lanes 1- 3- infected human. Lanes 4- 8 and 10- infected cat. Lane 9 - infected dog. + C- Control Positive is $M$. canis isolate (PTCC: 5069). - C - Control Negative is Trichophyton rubrum isolate(ATCC11404).

in $M$. canis DNA (Fig. 2, 3, 4). The relative risk to develop infection associated with the presence of SUBs, SUB2 (9/10) and SUB1 (8/10) seem to be higher in isolates obtained from dermatophytosis and SUB3 gene was observed in a low percentage (70\%; 7/10) of the isolates. Screening for the presence of SUB1-3 in isolates, representing $60 \%(6 / 10)$, were positive for SUBs, while in 10\% (1/10) none of the SUBs was observed (Table. 1). In comparing SCC to SDA for isolation of fungi, SCC as an inhibitory (Bacteria and Saprophytic fungi) medium is preferable to SDA for investigate of subtilisin genes presence in M. canis.

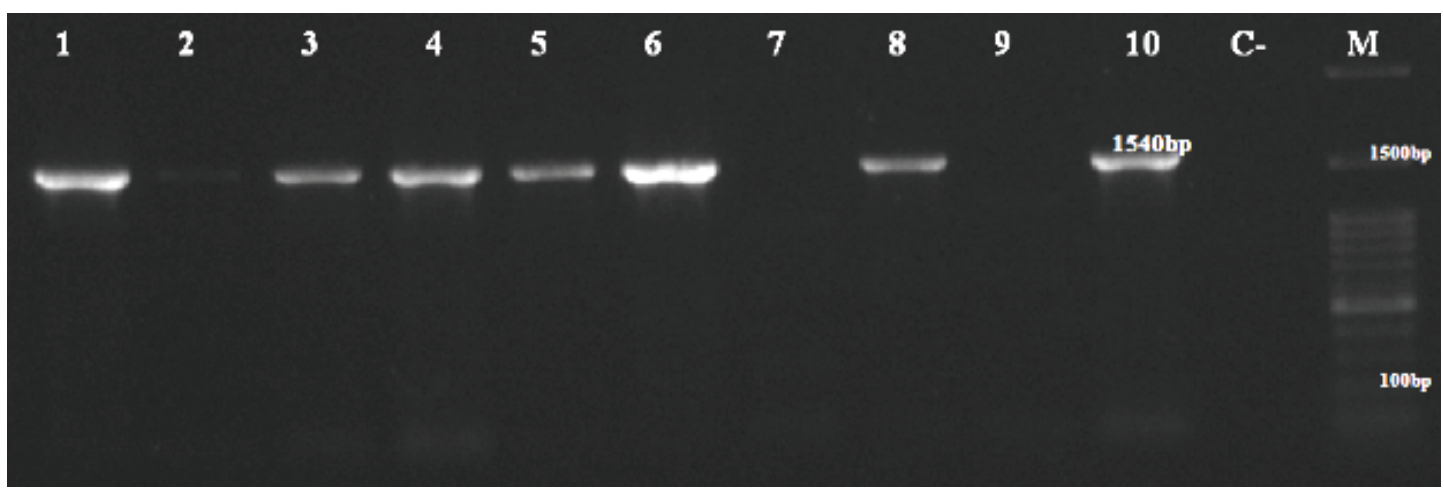

Figure 2: Positive results of 1540 bp fragment of SUB1 genes were obtained for 8/10 samples containing M. canis; Lanes 1- 3- infected human. Lanes 4- 8 and 10- infected cat. Lane 9 - infected dog. -C - Control Negative is Trichophyton rubrum isolate. Lane $\mathrm{M}-100-3000$ bp DNA molecular weight markers. 


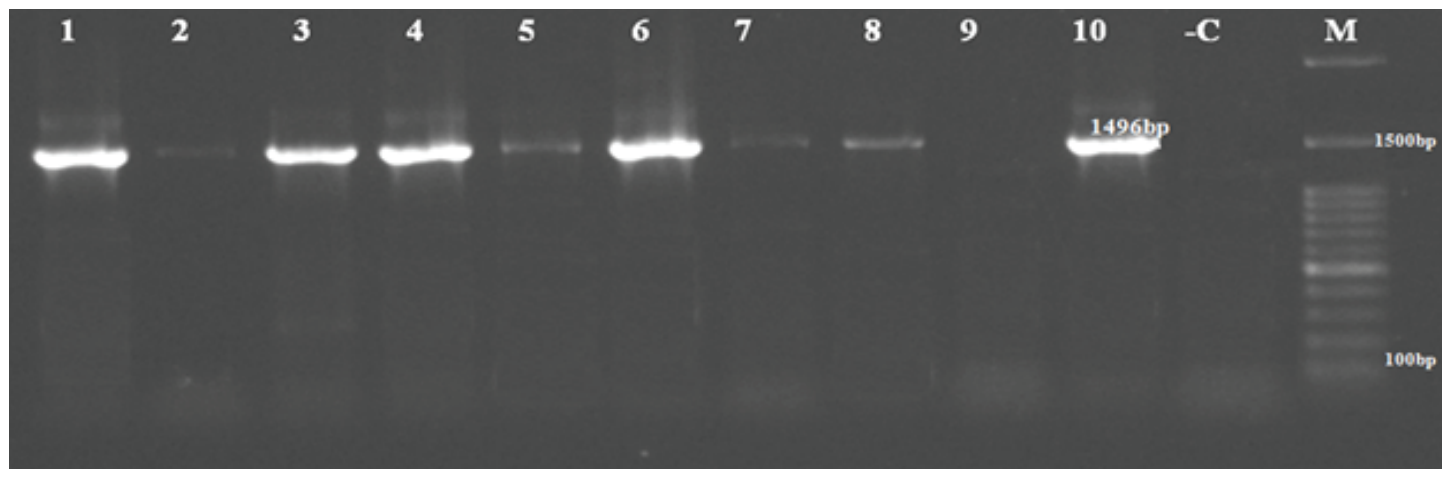

Figure 3: Positive results of 1496 bp fragment of SUB2 genes were obtained for 9/10 samples containing M. canis; Lanes 1- 3- infected human. Lanes 4- 8 and 10- infected cat. Lane 9 - infected dog. -C - Control Negative is Trichophyton rubrum isolate. Lane M-100 - 3000 bp DNA molecular weight markers.

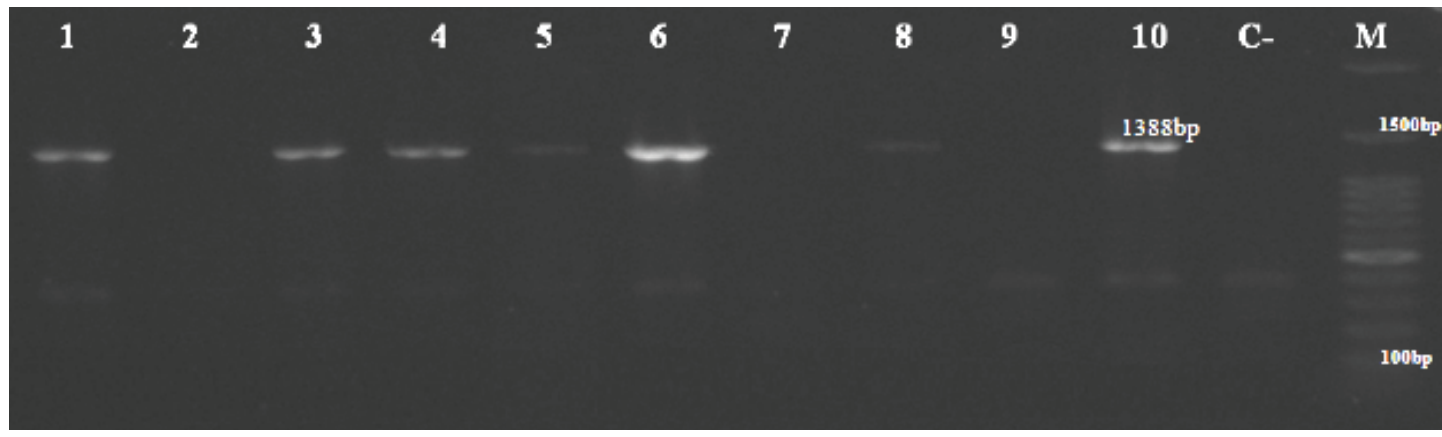

Figure 4: Positive results of 1388 bp fragment of SUB3 genes were obtained for $7 / 10$ samples containing M. canis; Lanes 1- 3- infected human. Lanes 4- 8 and 10- infected cat. Lane 9 - infected dog. -C - Control Negative is Trichophyton rubrum isolate. Lane $\mathrm{M}-100-3000$ bp DNA molecular weight markers.

TABLE 1: Distribution of SUBs on the analyzed Dermatophyte Infections.

\begin{tabular}{|c|c|c|c|c|c|c|}
\hline \multicolumn{7}{|c|}{ Hosts * SUBs Crosstabulation } \\
\hline \multirow{2}{*}{$\begin{array}{l}\text { Dermatophyte } \\
\text { Infections }\end{array}$} & & \multicolumn{4}{|c|}{ SUBs } & \multirow[t]{2}{*}{ Total } \\
\hline & & $\begin{array}{c}\text { SUB1 } \\
(n=8) \%\end{array}$ & $\begin{array}{l}\text { SUB2 } \\
(n=9) \%\end{array}$ & $\begin{array}{l}\text { SUB3 } \\
(n=7) \%\end{array}$ & $\begin{array}{c}\text { SUBs } \\
\text { absent(n=6)\% }\end{array}$ & \\
\hline \multirow[t]{2}{*}{ Hosts } & Human & 37.5 & 33.3 & 28.6 & 16.7 & 30.0 \\
\hline & Animal & 62.5 & 66.7 & 71.4 & 83.3 & 70.0 \\
\hline Total & & 100 & 100 & 100 & 100 & 100.0 \\
\hline
\end{tabular}

\section{Discussion}

fungal infections are one of the most common skin infections in humans and animals. Dermatophytes and saprophytic fungi are the most important fungal agents in the development of these infections [16]. M.canis serve as the zoophilic dermatophyte and its infection has considerable zoonotic importance that transmitted from pets to their owners diagnosed with dermatophytosis [17]. The most common isolated dermatophytes were M.canis by Cafarchia et al. (2006) in Bari, southern Italy [18]. Basiri et al. (2012) were 
reported the highest incidence of dermatophyte infection in dogs related to M.canis [19]. Katiraee et al. (2006) were investigated epidemiological factors of M.canis that transmitted from cats to their owners diagnosed with dermatophytosis [17]. Dermatophytes are pathogenic fungi that infect healthy people and the production of subtilisin proteases in vivo infection is known as an indicator of their importance in virulence of dermatophytes [20, 21]. A serine protease(SUBs) inhibitor, which significantly inhibits the adherence of $M$. canis arthroconidia to stratum corneum [22]. This study showed that a high percentage for SUB2 and SUB1virulence genes of $M$. canis isolates. Lemsaddek et al. (2010) were reported that SUB2 (82\%) and SUB1(79\%) detected in a high percentage of $M$. canis isolates [15]. At least the expression of SUB3 for keratinolytic activity in fungal infection is necessary [11]. SUB3 expression in $M$. canis DNA is a crucial event in initial contact $[23,24]$. We reported that SUB3 gene was observed in a low percentage. Lemsaddek et al. (2010) were reported that SUB3 detected in a low percentage (68\%) of M. canis isolates. These results suggested that SUB3 is not related to invade. In contrast, anti-SUB3 specific antibody $(\lg G)$

could be detected [11,25]. However, the expression of SUBs were shown during both adherence and early invasion [10]. In the present study, The absence of SUB 1, SUB2 and SUB3 in a M. canis isolated from dog with develop infection while in14\% none of the SUB genes was reported by Lemsaddek et al [15].

\section{Conclusion}

SUBs coding serine proteases on $M$. Canis give it the ability to digest keratinized tissues (skin and hair) in humans and animals and zoonotic attribute. In the present study, the absence SUBs in clinical isolate, indicates that they are indispensable for initial contact and adherence of fungi to host cells.

\section{Acknowledgements}

The data of this study were extracted from PhD thesis by Azadeh karami Robati and supported by Department of medical mycology in Tehran University and Veterinary University of Kerman, Iran.

\section{Funding}

This was self funded study. 


\section{Conflict of Interest}

None declared.

\section{Ethical Approval}

[All samples were collected using aseptic methods and in conformity with the ethical guidelines of the 1975 declaration of Helsinki].

\section{References}

[1] Cabañes F. Animal dermatophytosis. Recent advances. Revista Rev Iberoam Micol. 2000; 17(1): S8-12.

[2] Rezaei-Matehkolaei A, Makimura K, de Hoog GS, et al. Multilocus differentiation of the related dermatophytes Microsporum canis, Microsporum ferrugineum and Microsporum audouinii. Medic Microbiol 2012; 61(Pt 1):57-63.

[3] Hermoso de Mendoza M, Hermoso de Mendoza J, Alonso JM, et al. A zoonotic ringworm outbreak caused by a dysgonic strain of Microsporum canis from stray cats. Revista Iberoamericana De Micología. 2010; 27(2): 62-5.

[4] Achterman RR, White TC. Dermatophyte virulence factors: Identifying and analyzing genes that may contribute to chronic or acute skin infections. Int J Microbiol. 2012:358305.

[5] Scott DW, Miller WH, Griffin CE. Muller and Kirk's Small Animal Dermatology. 6th ed. Philadelphia, PA: WB Saunders; 2001. Structure and function of the skin; 26-27.

[6] Gumral R, Ilkit M. Turkish Journal of Medical Sciences Comparison of the contamination rates of culture media used for isolation and identification of dermatophytes. Turk J Med Sci. 2015; 45(3):587-92.

[7] Turska E, Konopka K, Turski W.The effect of chloramphenicol and cycloheximide on the activity of enzyme "markers" of mitochondrial substructures of the rat liver. Acta Biol Med Ger. 1977; 36(9):1231-6

[8] Mahler HR, Perlman P, Henson C. Selective effects of chloramphenicol, cycloheximide and nalidixic acid on the biosynthesis of respiratory enzymes in yeast Biochem Biophys Res Commun. 1968; 31(3): 474-80.

[9] Brillowska-Dabrowska A, Michalek E, Lindhardt Saunte DM, et al. PCR test for Microsporum canis identifi cation.. Med Mycol. 2013; 51:576-9.

[10] Baldoa A, Monod M, Mathy A, et al. Mechanisms of skin adherence and invasion by dermatophytes. Mycoses. 2012; 55(3):218-23. 
[11] Descamps F, Brouta F, Monod M, et al. Isolation of a Microsporum canis gene family encoding three subtilisin-like proteases expressed in vivo. J Invest Dermatol. 2002; 119(4):830-5.

[12] Chinnapun D.Virulence Factors Involved in Pathogenicity of Dermatophytes. Walailak J Sci \& Tech.2015; 12(7):573-80.

[13] Ayatollahi Mousavi SA, Karami Robati A, Madani M, et al. The study on rate of morbidity of fungal infections exiting in educational hospitals: Iran. Comp Clin Pathol. 2018; 27, 673-8.

[14] Karami Robati A, Khalili M, Hashemi Hazaveh SJ, et al. Assessment of the subtilisin genes in Trichophyton rubrum and Microsporum canis from dermatophytosis. Comp Clin Pathol. 2018; 1-5.

[15] Lemsaddek L, Chambel L, Tenreiro R. Incidence of fungalysin and subtilisin virulence genes in dermatophytes. In: Mendez A, editor. Current Research, Technology and Education Topics in Applied Microbiology and Microbial Biotechnology. Spain: FORMATEX; 2010. p 659-66.

[16] Mahmoudabadi AZ. A study of dermatophytosis in South West of Iran (Ahwaz). Mycopathol. 2005; 160(1): 21-4.

[17] Katiraee F, Javad Asharafi Helan J, Teifoori F. Multiple cases of feline dermatophytosis due to Microsporum canis transmitted to their owners. J Zoono Diseas. 2016; 1 (1):24-7.

[18] Cafarchia C, Romito D, Capelli G, et al. Isolation of microsporum canis from the hair coat of pet dogs and cats belonging to owners diagnosed with M. Canis tinea corporis. Veterin Dermatol. 2006; 17(5): 327-31.

[19] Basiri S, Ghazvini R, Hashemi SJ, et al. The Study On Fungal Isolates From Ownership Dogs With Skin Lesions In Rural Areas Of Meshkin-Shahr With Emphasize On Transmission Risk Of Fungal Zoonoses. J Payav Sala. 2015; 9 (3): 256-65.

[20] Scognamiglio T, Zinchuk R, Gumpeni P, et al. Comparison of inhibitory mold agar to Sabouraud dextrose agar as a primary medium for isolation of fungi. J Clini Microbio. 2010; 48(5): 1924-25.

[21] Brouta F, Descamps F, Monod M, et al. Secreted metalloprotease gene family of Microsporum canis. Infect Immun. 2002; 70(10):5676-83.

[22] Baldo A, Tabart J, Vermout $S$ et al. Secreted subtilisins of Microsporum canis are involved in adherence of arthroconidia to feline corneocytes. J Med Microbiol 2008; 57: 1152-6.

[23] Baldo A, Mathy A, Tabart J et al. Secreted subtilisin Sub3 from Microsporum canis is required for adherence to but not for invasion of the epidermis. Br J Dermatol 2010; 162: 990-7. 
[24] Vermout S, Tabart J, Baldo A et al. RNA silencing in the dermatophyte Microsporum canis. FEMS Microbiol Lett 2007; 275: 38-45.

[25] Deshmukh SK. Keratinophilic fungi isolated of Mumbai,. India Mycopathol. 2008; 146 : 115- 6. 